\title{
Lower incisor changes following non-extraction orthodontic decompensation in Class III surgical
} cases

\author{
Nor Nadia Zakaria ${ }^{1}$, Yasmin Kamarudin ${ }^{1 *}$, Ong Kah Shin ${ }^{2}$, Koo Zi Qing ${ }^{2}$
}

\section{KEYWORDS}

Orthognathic, decompensation, Class III malocclusion, lower incisor angle, Li-APo, prediction

\section{ABSTRACT}

The amount of incisor decompensation during pre-surgical orthodontics may affect the outcome of Class III orthognathic cases. The purpose of this study was to assess the lower incisor changes post-orthodontic decompensation in Class III surgical cases and to investigate the amount of crowding as a predictive factor. This was a retrospective study reporting on 22 Class III orthognathic cases. The lower incisor angulation (LIA) and distance of the lower incisor edge to the A-Pogonion line (Li-APo) were measured on pre-treatment and presurgical lateral cephalograms whereas crowding was measured on digitised pre-treatment study models. Pearson's correlation $(p<0.05)$ was used to assess the correlation of crowding with LIA and Li-APo changes, and prediction of the lower incisor decompensation was conducted using linear regression analysis. Results showed lower incisors were retroclined at $79.84^{\circ} \pm 7.08^{\circ}$ and positioned ahead of APo line by $6.52 \mathrm{~mm} \pm 2.97$ $\mathrm{mm}$ at the start of treatment. Pre-surgical LIA and Li-APo were found to increase following orthodontic decompensation to $90.43^{\circ} \pm 5.96^{\circ}$ and $10.34 \mathrm{~mm} \pm 3.25 \mathrm{~mm}$, respectively. There was a moderate positive correlation ( $r=0.592$ ) between crowding and Li-APo changes which was statistically significant, $p$ value $=0.004$, and had a strong predictor with $31.8 \%$ predictability. However, LIA showed a weak correlation $(r=0.329)$ with crowding and was not statistically significant $(p=0.135)$. Li-APo changes during orthodontic decompensation can be predicted with $31.8 \%$ predictability using the formula; Li-APo change $=2.064+0.503$ (crowding) .

\section{INTRODUCTION}

There are a few approaches to treating a Class III malocclusion but in severe cases where skeletal deformity is too great to be masked by orthodontic camouflage alone, orthognathic surgery is recommended. It involves surgical repositioning of the maxilla, mandible and dentoalveolar segments to achieve occlusal and facial harmony [1]. Skeletal Class III patients generally display marked proclination of upper incisors and retroclination of

\footnotetext{
${ }^{1}$ Department of Paediatric Dentistry and Orthodontics, Faculty of Dentistry, Universiti Malaya, Kuala Lumpur 50603, Malaysia. ${ }^{2}$ Faculty of Dentistry, Universiti Malaya, Kuala Lumpur 50603, Malaysia.

*Correspondence: yasminkamarudin@um.edu.my
}

lower incisors. This is nature's way to attempt to achieve a harmonious inter-arch relationship to compensate for the underlying skeletal discrepancies, a phenomenon known as dentoalveolar compensation [2]. Patients who require combined orthodontic-orthognathic approach will undergo pre-surgical orthodontic treatment to remove this natural compensation (orthodontic decompensation) which is then followed by orthognathic surgery.

Adequate pre-surgical incisor decompensation is important as it influences the magnitude of surgical movements and this will affect the surgical outcome [3]. Dental decompensation planning also depends on the anteroposterior and vertical skeletal pattern. Aligning the teeth back to their ideal axial inclination usually leaves the patient 
looking worse temporarily, with an increase in the reverse overjet. However, this step is necessary so that both the maxillary and mandibular arches will coordinate after orthognathic surgery [4]. For arch alignment to occur, crowding must be resolved by creation of space. Crowding is defined as a discrepancy between tooth size and jaw size [5]. Space creation may be achieved by tooth extractions, molar distalisation, proximal tooth reduction, expansion, or proclination of the incisors. In non-extraction cases, space gain in the lower arch is mainly achieved from uprighting of buccal segments and arch lengthening via lower incisor proclination and protrusion.

This study aimed to assess the changes in lower incisors following decompensation in nonextraction Class III surgical patients by measuring the anteroposterior position of the lower incisor edges, and changes in the lower incisor angulation after completion of orthodontic decompensation. This study also aimed to investigate the relationship between crowding and lower incisor changes upon orthodontic decompensation, and to predict the changes of lower incisor based on the amount of crowding. The percentage of adequately decompensated teeth in preparation for orthognathic surgery was also evaluated. The study was limited to non-extraction cases to reduce confounding factors that could influence the lower incisor changes.

\section{MATERIALS AND METHODS}

Following approval by the Medical Ethics Committee, Faculty of Dentistry (Reference number DF CD 1904/0031(U)), this retrospective study was conducted on records of Class III orthognathic surgical patients following orthodontic decompensation. Samples were recruited from a population of patients from the Department of Paediatric Dentistry and Orthodontics, and the Department of Oral and Maxillofacial Clinical Sciences, Faculty of Dentistry, Universiti Malaya from 2005 to 2020.

The inclusion criteria included patients who presented with Class III malocclusion with underlying skeletal discrepancy and had undergone or were scheduled for orthognathic surgery to correct the malocclusion; cases with non-extraction in the lower arch (excluding third molars); patients who had completed pre-surgical orthodontic decompensation; and cases with complete records which included initial study models and both pretreatment and pre-surgical lateral cephalograms.
The exclusion criteria were cases with craniofacial deformities or clefting; patients with a history of hypodontia in the lower arch, previously extracted teeth or required extractions of permanent mandibular teeth as part of their orthodontic treatment; cases with no or incomplete sets of initial study models and lateral cephalograms.

A total of 43 cases were screened. Cephalometric measurements were done to assess the lower incisor changes following orthodontic decompensation prior to orthognathic surgery. The lower incisor changes were studied by measuring the anteroposterior position of the lower incisor edge and the lower incisor angulation at the pretreatment and pre-surgical stages. The effects of crowding on the lower incisor changes were also studied, and this was used to predict the changes of lower incisors based on the amount of crowding. The percentage of adequately decompensated incisors in preparation for orthognathic surgery was also evaluated by comparing to average racial norms.

\section{Measurement of lower incisor changes}

Lateral cephalograms taken pre-treatment and presurgery (upon completion of pre-surgical orthodontic treatment) were traced and analysed using OPAL 2.4 (British Orthodontic Society, London, UK), a digital cephalometric tracing program. Magnification, alteration of brightness, and contrast tools were used to improve accuracy of tracing the cephalograms. The distance between the lower incisor edge to the line which extends from Point $A$ to Pogonion (Li-APo) was used to determine the protrusion of the lower arch. The lower incisor angulation (LIA) in relation to the mandibular plane was measured to determine the axial inclination of mandibular incisors (Figure 1).

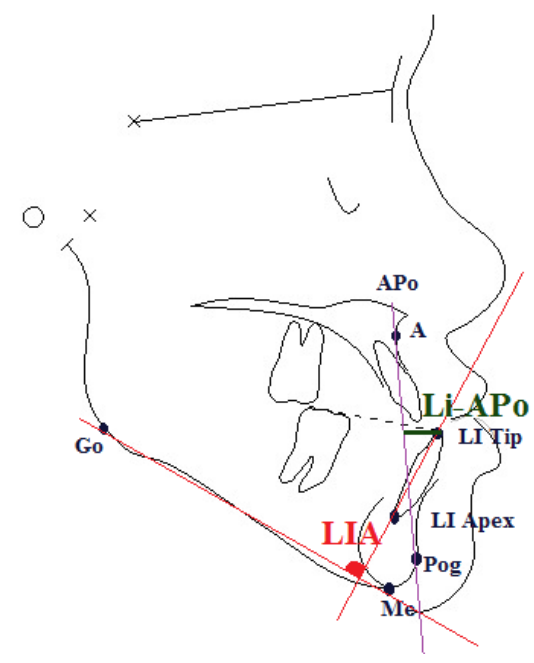

Figure 1 Illustration of Li-APo and LIA 
The amount of decompensation achieved was determined by comparing the LIA and Li-APo during the pre- and post-orthodontic decompensation. Pre-surgical LIA were compared to racial norms to determine if the cases were adequately decompensated prior to surgery. Considering the normal range of LIA differs in each ethnicity and race, different racial norms were used based on the patient's ethnicity. The normal range of LIA in the Malay ethnic group was set at $97^{\circ} \pm 6^{\circ}[6]$, Chinese ethnic group at $95^{\circ} \pm 5^{\circ}$, and $93^{\circ} \pm 6^{\circ}$ for Indian ethnic group [7].

The cephalometric tracings were done by a single examiner (OKS), who was trained and calibrated against an experienced orthodontist (YK). Ten lateral cephalograms were measured and interexaminer reliability was assessed using system Intraclass Correlation Coefficient (ICC). Measurements were repeated after two weeks to assess intra-examiner reliability.

\section{Measurement of pre-treatment crowding}

Pre-treatment mandibular study models were scanned using Maestro 3D scanner MDS200 (AGE Solutions S.r.I., Italy) and Maestro 3D Easy Dental Scan software (AGE Solutions S.r.I., Italy) (Figure 2). Measurements of the digitised models were then carried out using Maestro Ortho Studio software (AGE Solutions S.r.I., Italy).

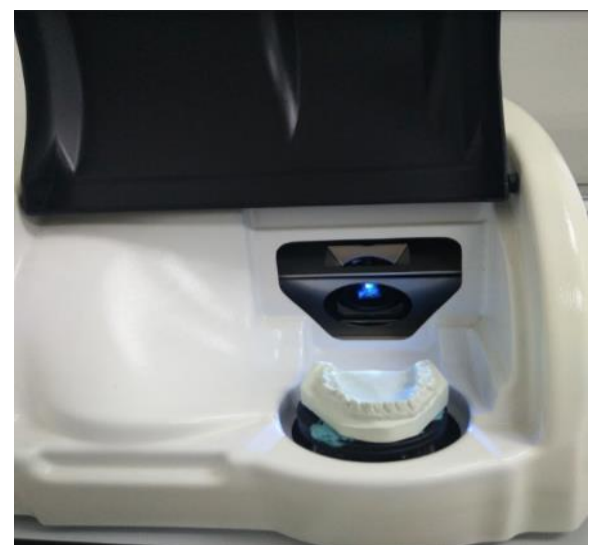

Figure 2 Study model scanning using Maestro 3D scanner

Crowding occurs when the combined mesio-distal tooth width exceeds the available space within the arch. This was measured by subtracting the total width of the teeth present (total arch width) from the total amount of space available (arch length). Arch length, also known as arch perimeter, is the combined distance between the mesial anatomic contact points of bilateral lower first permanent molars to the contact point between the mandibular central incisors [8]. In this study, the
Lundstrom segmental method was used to measure the arch length. The study models were orientated and viewed from the occlusal view. Linear sections of the arch were measured bilaterally from mesial of the first permanent molar to mesial of the first premolar, and from mesial of the first premolar to mesial of the central incisor. The sum of the 4 sections was then calculated to determine the arch length (Figure 3).

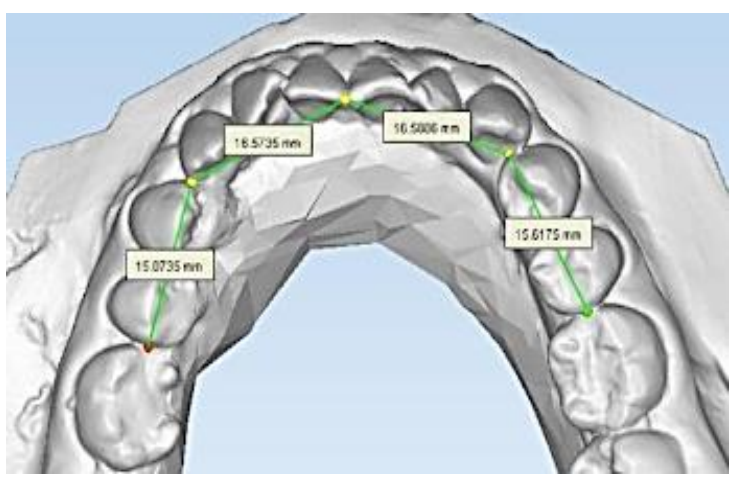

Figure 3 Measurement of arch length (segmental method) using Maestro Ortho Studio software

To calculate the total arch width, the widest mesiodistal diameter [8] of each individual permanent tooth from the second premolar on the left quadrant to the second premolar on the right quadrant was measured using the software's measurement tool, accurate to the nearest 0.00001 $\mathrm{mm}$ (Figure 4). The sum of all the tooth widths was then calculated. Next, the total arch width was subtracted from the total arch length to determine the amount of crowding. Negative values indicated crowding, while positive values indicated spacing.

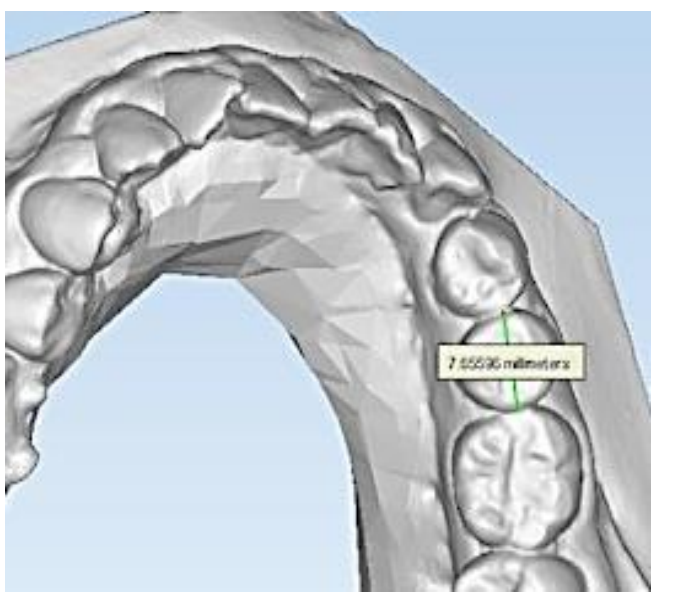

Figure 4 Measurement of tooth width using Maestro Ortho Studio software

All values were rounded off to two decimal places. The measurements were done by a single examiner (KZQ), who was trained and calibrated against YK. Ten dental casts were measured, and interexaminer reliability was assessed using system Intraclass Correlation Coefficient (ICC). 
Measurements were repeated after two weeks to assess intra-examiner reliability.

Statistical package for the social sciences (SPSS) software version 23.0.1 for Windows (Armonk, NY: IBM Corp.) was used for statistical analysis. Normality test (Shapiro-Wilk) was used to determine if the results were normally distributed. The correlation of crowding with lower incisor decompensation (Li-APo and LIA readings) were determined using Pearson's correlation test, and prediction of the lower incisor decompensation based on the amount of crowding was conducted using linear regression analysis. The size interpretation of a correlation coefficient by Evans (1996) was used as reference [9]. Level of significance was set at $p<0.05$.

\section{RESULTS}

Both intra- and inter-examiner reliability tests were assessed using the system ICC. Based on the 95\% confidence interval of the ICC estimate, values less than 0.5 is poor, between 0.5 and 0.75 is moderate, between 0.75 and 0.9 is good, and greater than 0.90 is indicative of excellent reliability [10]. The ICC values of 0.820-0.966 in this study indicated good to excellent reliability for both intra- and interexaminer reliability.

A total of 22 cases that fulfilled the inclusion criteria were selected out of 43 cases, comprising of 11 Malay (50\%), 10 Chinese (45.45\%), and one Indian (4.54\%). The mean age was $24.36 \pm 4.98$ with 17 females and 5 males. The test of normality (ShapiroWilk) was done and data was found to be normally distributed. Table 1 shows descriptive analysis of LIA, Li-APo and crowding values.

The findings showed that the lower incisor edge lay on average $6.52 \mathrm{~mm}$ ahead of the APo line at the pre-treatment stage. It was then advanced on average about $3.82 \mathrm{~mm}$ after orthodontic decompensation. The pre-treatment LIA had a mean value of $79.84^{\circ}$, which increased to a mean value of $90.43^{\circ}$ at the pre-surgical stage, with a mean difference of $10.59^{\circ}$. The mean value of crowding was measured at $3.49 \mathrm{~mm}$ at the start of treatment.

Pearson correlation coefficient was used to assess the relationship between crowding and LIA changes (Figure 5) and results indicated a weak positive correlation between the two variables and was not statistically significant, $r(1)=0.329, p=0.135$.

Pearson correlation coefficient was used to assess the relationship between crowding and Li-APo (Figure 6) and results indicated a moderate positive correlation between the two variables and was statistically significant, $r(1)=0.592, p=0.004$.

Table 1 Descriptive statistics of Li-APo, LIA and crowding in pre-treatment and pre-surgical stage $(n=22)$

\begin{tabular}{rcccc}
\hline & Min & Max & Mean & SD \\
\hline${ }^{\mathrm{a}} 1$ & -0.05 & 11.00 & 6.52 & 2.97 \\
\hline${ }^{\mathrm{a}} 2$ & 3.50 & 17.50 & 10.34 & 3.25 \\
\hline${ }^{\mathrm{a}} 3$ & 3.55 & 6.50 & 3.82 & 0.28 \\
\hline${ }^{\mathrm{a}} 4$ & 66.00 & 93.00 & 79.84 & 7.08 \\
\hline${ }^{\mathrm{a}} 5$ & 82.00 & 105.00 & 90.43 & 5.96 \\
\hline${ }^{\mathrm{a}} 6$ & 16.00 & 12.00 & 10.59 & -1.12 \\
\hline${ }^{\mathrm{a}} 7$ & $-1.60^{*}$ & 13.35 & 3.49 & 3.74
\end{tabular}

1: Pre-treatment Li-APo $(\mathrm{mm}) ; 2$ : Pre-surgical LiAPo $(\mathrm{mm})$; 3: Li-Apo change $(\mathrm{mm})$; 4: Pre-treatment LIA $\left({ }^{\circ}\right)$; 5: Pre-surgical LIA $\left({ }^{\circ}\right)$; 6: LIA change $\left({ }^{\circ}\right)$; 7 : Crowding ( $\mathrm{mm}$ )

*Indicates spacing

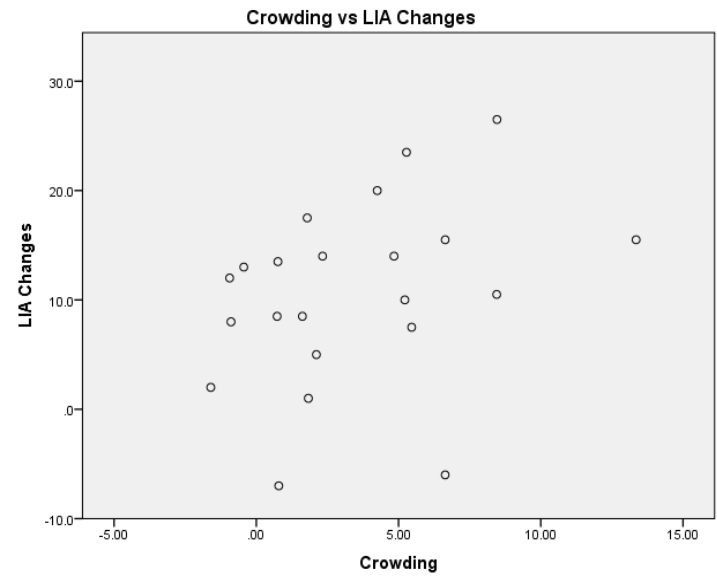

Figure 5 Scatter plot of crowding and LIA changes

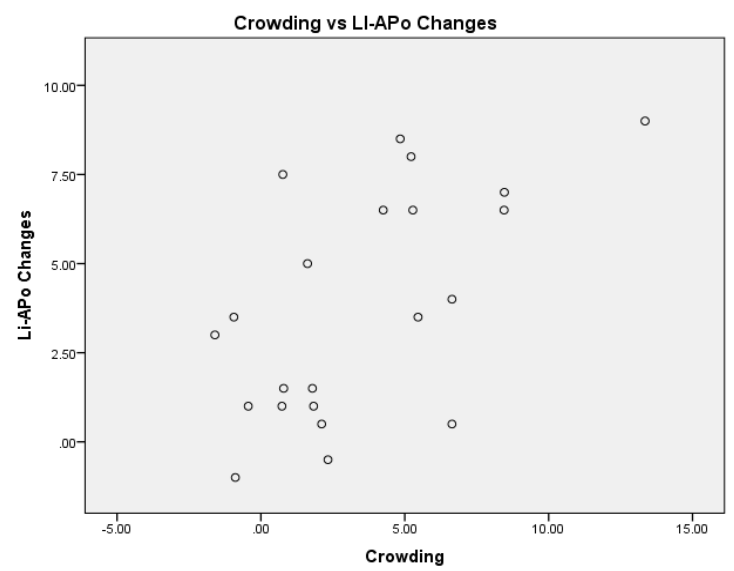

Figure 6 Scatter plot of crowding and Li-APo changes 
Further analysis was conducted to assess the predictive effects of crowding towards the Li-APo. This was achieved using Linear Regression where the earlier predictor was assessed as statistically significant. Using the Enter method, the results revealed that crowding affects the range of Li-APo $(p=0.004)$ with the $\mathrm{F}=10.791$, Beta coefficient $=$ 2.064, $R=0.592$ and $R^{2}=0.318$. This means that crowding can explain $31.8 \%$ of variations in the results when using the formula, Li-APo change = $2.064+0.503$ (crowding).

Out of 22 patients studied, only 14 patients (63.63\%) were adequately decompensated to the average range of $\mathrm{LIA}$, according to their respective race (Table 2 ). The mean for the adequately decompensated cases was $93.93^{\circ} \pm 6.83^{\circ}$ (Malay) and $92.23^{\circ} \pm 2.73^{\circ}$ (Chinese) as compared to the inadequately decompensated cases with $86.75^{\circ} \pm$ $1.55^{\circ}$ (Malay) and $83.38^{\circ} \pm 1.80^{\circ}$ (Chinese).

Table 2 Percentage of cases with adequately decompensation lower incisors

\begin{tabular}{lcc}
\hline & $\begin{array}{c}\text { Adequately } \\
\text { decompensated }\end{array}$ & $\begin{array}{c}\text { Inadequately } \\
\text { decompensated }\end{array}$ \\
\hline $\begin{array}{l}\text { No of } \\
\text { patients }\end{array}$ & 14 & 8 \\
\hline$\%$ & 63.63 & 36.37 \\
\hline
\end{tabular}

\section{DISCUSSION}

Orthodontic decompensation in skeletal Class III malocclusion aims to correct the axial inclination of the lower incisors by reversing the natural dental compensation. The amount of mandibular incisor decompensation may influence the magnitude of surgical movements that can be achieved, and thus the surgical outcome [3]. As a rule of thumb, 5 degrees of incisor proclination will result in $1 \mathrm{~mm}$ of space [11], and $1 \mathrm{~mm}$ of incisor tip advancement will result in $1 \mathrm{~mm}$ of space [12]. This formula has been used when predicting the amount of incisor decompensation during treatment planning. However, this method only applies for the upper arch, where the incisors are of average shape and size, and should not be used for the lower incisors. As of yet, we are not aware of any formula which is available for predicting the amount of lower incisor changes due to crowding. In this study, both the anteroposterior position of the lower incisor edges and LIA increased following orthodontic decompensation. This is expected as lower incisors are usually retroclined in Class III malocclusions due to the natural dental compensation. The built-in prescription of orthodontic brackets will cause proclination of the lower incisors and forward movement of the incisal edges. This finding is in accordance with other studies of surgical Class III cases [13], [2]. In this study, cases were limited to non-extraction cases, and thus space was gained mostly by proclining the lower incisors and uprighting of the lingually inclined buccal segments.

The relationship between crowding and lower incisor decompensation was investigated using Pearson's correlation. The results showed crowding had a moderate positive correlation that was statistically significant, and a weak positive correlation that was not statistically significant with Li-APo and LIA, respectively. This means there is a weak relationship between crowded mandibular dentition with LIA after decompensation. However, the relationship of crowding with Li-APo was statistically significant, indicating that crowding could influence the anteroposterior movement of the lower incisors. It is unclear why only linear measurements of the lower incisors was correlated to crowding. This could be explained by in-built torque values within the bracket slots that may limit the amount of changes in incisor inclination. Treatment mechanics such as Class II elastics may also favour more incisor protrusion as opposed to incisor inclination change. Further analysis using Linear Regression was conducted and it was revealed that crowding is a strong predictor with $31.8 \%$ predictability towards Li-APo changes. This means the study does have clinical implication, as clinicians are able to predict the amount of dental decompensation that can be achieved prior to orthognathic surgery using the following formula, Li-APo change $=2.064+0.503$ (crowding). The ability to predict the amount of lower incisor decompensation prior to commencing treatment may aid in treatment planning, especially in terms of determining the type and magnitude of surgical movements. The accuracy of this formula, however, is yet to be tested.

Pre-surgical orthodontics aims to establish the correct lower incisor position to aid in surgical movements. Patients who have adequately decompensated lower incisors have larger surgical movements, and present post-operative cephalometric values that are more compatible with average values [3]. Inadequate incisor positioning can compromise buccal interdigitation, increase the surgical morbidity, and compromise the facial aesthetics and stability [14]. Insufficient reverse overjet preparation can limit the magnitude of maxillary advancement or mandibular setback in achieving a Class I skeletal base during surgery. In our study, only $64 \%$ of the subjects were 
adequately decompensated which was lesser than other reported studies [15-16].

In some cases, a conscious decision is made to limit the amount of decompensation achieved presurgical, due to dental and periodontal health concerns. Attempts to fully correct the lower incisor inclination in severely retroclined lower incisors could jeopardise the integrity of the periodontal tissue although some studies have reported no association between the two parameters [17-18]. This is particularly true in patients presenting with thin gingival phenotype [19]. Other reasons for inadequate decompensations include inadequate labial bone and lack of periodontal support to allow for sufficient advancement of the incisors, lower lip neuromuscular resistance to mandibular incisor advancement, and poor patient compliance with intraoral elastic traction [20]. It has also been suggested that the morphology of the symphysis might affect incisor movement in patients with mandibular prognathism [21]. In cases with severe skeletal discrepancy, the decision to not fully decompensate the incisors may be made to limit the magnitude of surgical movements, thus improving stability and predictability of the surgical outcome. These variables, however, were not investigated in this study.

Digital measurements of crowding and lateral cephalometric radiographs were used. Although the Lundstrom segmental approach used to measure arch length in this study has potential to overestimate crowding due to the curvature of the archform, no statistical difference between the measurements of crowding using the Nance brass wire or segmental method has been reported [8]. Hence, the segmental method was deemed appropriate and more convenient as it eliminates the subjectivity of identifying the arch perimeter. Studies have shown that measurements using relevant software on digitised study models were reproducible to linear measurements using the conventional methods of digital callipers on plaster models [22]. Digital lateral cephalogram tracing was used in this study to reduce human errors associated with hand tracing. A point and lower incisor apex are notoriously difficult to identify and have an increased envelope of error due to overlapping structures and poor quality of radiographic image [23]. To improve the accuracy of landmark identification, magnification and contrast tools in the OPAL 2.4 software were used. The overall differences in landmark identification between the conventional and digitised radiographs, have been shown to be statistically significant, as the latter provides higher reliability in landmark identification, which implies a comparable reliability of the subsequently derived cephalometric measurements [24].

Overall, this study had some limitations due to its small sample size and samples were recruited from a single centre, thus limiting the generalisability of the study outcomes. A larger sample size that includes multicentre sampling is recommended in future studies, with attempts to explore other confounding factors that may influence the lower incisor changes during treatment. The use of three dimensional imaging to aid in assessing the bone quality and its impact on decompensation, dehiscence and fenestrations of teeth needs to be explored in severe Class III malocclusion. Cases that do not intend to be fully decompensated for various reasons as stated during the treatment planning stage, may be eliminated to have a more accurate measurement of the overall success rate based on planned tooth movement instead of racial norms.

\section{CONCLUSION}

The following conclusions are drawn from our study on lower incisor changes following orthodontic decompensation:

1. The lower incisors proclined at an average of $10.59^{\circ} \pm 8.27^{\circ}$ following orthodontic decompensation.

2. The lower incisors advanced $3.82 \mathrm{~mm} \pm 3.18 \mathrm{~mm}$ following orthodontic decompensation.

3. Crowding had a moderate positive linear relationship with Li-APo changes and had a strong predictor with $31.8 \%$ predictability.

4. The success rate of orthodontic decompensation of lower incisors in Class III surgical cases was $63.63 \%$.

\section{ACKNOWLEDGEMENT}

The authors would like to thank the Department of Paediatric Dentistry and Orthodontics and Department of Oral and Maxillofacial Clinical Sciences for their support and Biomaterial Research Laboratory for the assistance in conducting this research.

\section{DECLARATION OF INTEREST}

The authors report no conflict of interest. The authors alone are responsible for the content of this article. 


\section{REFERENCES}

1. Littlewood SJ, Mitchell L. An Introduction to Orthodontics. $5^{\text {th }}$ ed. Oxford: Oxford University Press; 2019.

2. Kim SJ, Kim KH, Yu HS, Baik HS. Dentoalveolar compensation according to skeletal discrepancy and overjet in skeletal Class III patients. Am J Orthod Dentofacial Orthop. 2014;145(3):317-24.

3. Capelozza Filho L, Martins A, Mazzotini R, da Silva Filho OG. Effects of dental decompensation on the surgical treatment of mandibular prognathism. Int J Adult Orthodon Orthognath Surg. 1996;11(2):165-80.

4. McIntyre GT. Treatment planning in Class III malocclusion. Dent Update. 2004;31(1):13-20.

5. Hussain SS, Ashraf B, Khan SQ. Relationship of dental crowding to tooth size and arch dimensions in class I normal \& class I malocclusion sample. Pak Oral Dental J. 2014;34(4).

6. Hassan MS. Cephalometric Norm for Malay. Universiti Malaya. 1998. (unpublished)

7. Adnan and Hussain. Cephalometric Norm for Chinese and Indian. Universiti Malaya. 1998. (unpublished)

8. Suja AG, Babu EC, Presanth SS. Assessment of dental crowding. Int J Basic Appl Med Sci. 2014;4(1):52-55.

9. Evans JD. Straightforward Statistics for the Behavioral Sciences. Thomson Brooks/Cole Publishing Co; 1996.

10. Koo TK, Li MY. A Guideline of Selecting and Reporting Intraclass Correlation Coefficients for Reliability Research. J Chiropr Med. 2016;15(2):155-63.

11. O'Higgins EA, Kirschen RH, Lee RT. The influence of maxillary incisor inclination on arch length. Br J Orthod. 1999;26(2):97-102.

12. O'Higgins EA, Lee RT. How much space is created from expansion or premolar extraction? J Orthod. 2000;27:11-13.

13. Troy BA, Shanker S, Fields HW, Vig K, Johnston W. Comparison of incisor inclination in patients with Class III malocclusion treated with orthognathic surgery or orthodontic camouflage. Am J Orthod Dentofacial Orthop. 2009;135(2):146-e1.

14. Miloro M, Ghali GE, Larson P, Waite P. Peterson's Principles of Oral and Maxillofacial Surgery. $3^{\text {rd }}$ ed. Connecticut: People's Medical Publishing House; 2011.

15. McNeil C, McIntyre GT, Laverick S. How much incisor decompensation is achieved prior to orthognathic surgery? J Clin Exp Dent. 2014;6(3):e225.

16. Ahn HW, Baek SH. Skeletal anteroposterior discrepancy and vertical type effects on lower incisor preoperative decompensation and postoperative compensation in skeletal Class III patients. Angle Orthod. 2011;81(1):64-74.

17. Allais D, Melsen B. Does labial movement of lower incisors influence the level of the gingival margin? A casecontrol study of adult orthodontic patients. Eur J Orthod. 2003;25(4):343-52.

18. Choi YJ, Chung CJ, Kim KH. Periodontal consequences of mandibular incisor proclination during presurgical orthodontic treatment in Class III malocclusion patients. Angle Orthod. 2015;85(3):427-33.

19. Melsen B, Allais D. Factors of importance for the development of dehiscences during labial movement of mandibular incisors: a retrospective study of adult orthodontic patients. Am J Orthod Dentofacial Orthop. 2005;127(5):552-61.

20. Johnston C, Burden D, Kennedy D, Harradine N, Stevenson M. Class III surgical-orthodontic treatment: a cephalometric study. Am J Orthod Dentofacial Orthop. 2006;130(3):300-9.

21. Ricketts RM. The Keystone Triad. Am J Orthod Dentofacial Orthop. 1964;50(4):244-64.

22. Julku J, Hannula M, Pirilä-Parkkinen K, Tolvanen M, Pirttiniemi P. Dental arch effects after early and later timed cervical headgear treatment-a randomized controlled trial. Eur J Orthod. 2019;41(6):622-30.

23. Durão AP, Morosolli A, Pittayapat P, Bolstad N, Ferreira AP, Jacobs R. Cephalometric landmark variability among orthodontists and dentomaxillofacial radiologists: a comparative study. Imaging Sci Dent. 2015;45(4):213-20.

24. Chen YJ, Chen SK, Chung-Chen Yao J, Chang HF. The effects of differences in landmark identification on the cephalometric measurements in traditional versus digitized cephalometry. Angle Orthod. 2004;74(2):15561. 


\section{Editorial History}

Date of Submission: 8 Dec 2020

Review \& Revision: 14 Dec 2020 - 9 Feb 2021

Accepted: 16 Feb 2021

Published: 3 March 2021

License Information: This work is licensed under a Creative Commons Attribution 